\title{
Caractérisation physico-chimique des horizons de surface de sols à maraîchers dans la ville d'Abidjan (Côte d'Ivoire)
}

\author{
Kouassi Joseph KOUAKOU ${ }^{1 *}$, Seu Jonathan GOGBEU ${ }^{2}$, Ahoua Edmond SIKA ${ }^{3}$, \\ Koffi Bertin YAO ${ }^{1}$, Moussa BOUNAKHLA ${ }^{4}$, Fatiha ZAHRY ${ }^{4}$, Mounia TAHRI ${ }^{4}$, \\ Denezon Odette DOGBO $^{1}$ et Yves-Alain BEKRO
}

\author{
${ }^{1}$ Université Nangui Abrogoua, Unité de Formation et de Recherche des Sciences de la Nature, Laboratoire de \\ Biologie et Amélioration des Productions Végétales, 02 BP 801 Abidjan 02, Côte d'Ivoire. \\ ${ }^{2}$ Université Jean Lorougnon Guédé, Laboratoire de Physiologie et Pathologie Végétales, BP 150 Daloa, \\ Côte d'Tvoire. \\ ${ }^{3}$ Université Nangui Abrogoua, Unité de Formation et de Recherche des Sciences et Gestion de \\ l'Environnement, 02 BP 801 Abidjan 02, Côte d'Ivoire. \\ ${ }^{4}$ Laboratoire d'Analyses Elémentaires, Centre National de l'Energie, des Sciences et des Techniques \\ Nucléaires, BP 1382 R.P. 10001 Rabat, Maroc. \\ ${ }^{5}$ Université Nangui Abrogoua, Unité de Formation et de Recherche des Sciences Fondamentales et Appliquées, \\ Laboratoire de Chimie Bio-Organique et des Substances Naturelles, 02 BP 801 Abidjan 02, Côte d'Ivoire. \\ *Auteur correspondant; E-mail : ecologue12@yahoo.fr
}

\section{RESUME}

A l'instar des grandes agglomérations africaines, le maraîchage est en pleine expansion dans la ville d'Abidjan (Côte d'Ivoire). Les sols à maraîchers sont des réceptacles de déchets de toutes natures qui pourraient modifier leurs propriétés agro-pédologiques et leurs teneurs en éléments traces. Le but de ce travail est d'identifier, au moyen d'une analyse en composantes principales, les paramètres agro-pédologiques et les éléments traces qui caractérisent les horizons de surface de sols à maraîchers dans la ville d'Abidjan. L'étude a été menée sur la ferme expérimentale de l'Université Nangui Abrogoua et deux sites de maraîchage, à savoir, Marcory et Cocody. Les résultats ont révélé que les sols à Marcory sont caractérisés par les teneurs les plus élevées en zinc, en cuivre et en matière organique. Par contre, ceux de Cocody sont plus riches en nickel, en cadmium et en plomb, tandis que le manganèse, le fer, le nickel et l'argile ont les teneurs les plus élevées dans les sols de la parcelle expérimentale de l'université.

(C) 2019 International Formulae Group. All rights reserved

Mots clés: Analyse en composantes principales, propriétés agro-pédologiques, éléments traces, sols à maraîchers, Abidjan.

\section{Physico-chemical characterization of superficial horizons from market garden soils in the Abidjan city (Côte d'Ivoire)}

\section{ABSTRACT}

Like the big African agglomerations, market gardening is booming in the Abidjan city (Côte d'Ivoire). Market gardening soils are receptacles of all types of waste that could modify their agro-pedological properties 
and their trace element contents. The purpose of this work is to identify, through a principal component analysis, the agro-pedological parameters and the trace elements that characterize the surperficial horizons of market garden soils in the Abidjan city. The study was conducted on the experimental farm of Nangui Abrogoua University and two market gardening sites, namely, Marcory and Cocody. The results revealed that Marcory soils are characterized by the highest levels of zinc, copper and organic matter. On the other hand, those of Cocody are richer in nickel, cadmium and lead, whereas the manganese, the iron, the nickel and the clay have the highest contents in the grounds of the experimental plot of the university.

(C) 2019 International Formulae Group. All rights reserved

Keywords: principal components analysis, agro-pedological properties, trace elements, market garden soils, Abidjan.

\section{INTRODUCTION}

La plupart des sols urbains cultivés sont des réceptacles privilégiés des effluents industriels, des eaux usagées des activités domestiques et des retombées atmosphériques. D'autres déchets de toutes natures sont également susceptibles d'être épandus sur ces sols urbains cultivés ou bien l'ont été dans le passé (composts d'ordures ménagères, boues d'industries, boues de papeteries, etc). Ces différentes pratiques peuvent modifier les propriétés physiques, chimiques et biologiques des sols (Baize, 2000). Parmi les éléments indésirables contenus dans les divers déchets, figurent les éléments traces métalliques (ETM), appelés communément métaux lourds, potentiellement toxiques pour l'Homme, les animaux et les plantes. Des apports d'éléments traces sont, également, dûs aux pratiques agricoles classiques (fertilisants, chaulages, fumiers, traitements phytosanitaires) (Baize, 2000). La pollution par ces éléments entraîne leur accumulation dans les sols (Tremel-Schaub et Feix, 2005) et, sous certaines conditions, leur transfert du sol à la plante est inévitable. C'est pourquoi, l'agriculture urbaine fait l'objet de larges controverses en matière de sécurité alimentaire (Kouakou et al., 2008).

A l'instar des grandes agglomérations africaines, le marâichage est en pleine expansion dans la ville d'Abidjan et les sols à maraîchers sont fertilisés avec de la fiente de volaille, source de matière organique, d'éléments nutritifs et d'éléments traces métalliques potentiellement toxiques (Kouakou et al., 2008).
Le but de ce travail est d'identifier les paramètres agro-pédologiques et les éléments traces qui caractérisent les horizons de surface de sols à maraîchers dans la ville d'Abidjan (Côte d'Ivoire), en vue de savoir si les sols des sites à l'étude sont semblables ou dissemblables pour les paramètres étudiés.

\section{MATERIEL ET METHODES}

\section{Sites de prélèvement des sols}

L'agglomération d'Abidjan est située au Sud-Est de la Côte d'Ivoire et est comprise entre les latitudes $5^{\circ} 00^{\prime}$ et $5^{\circ} 30^{\prime}$ nord et les longitudes $3^{\circ} 50^{\prime}$ et $4^{\circ} 10^{\prime}$ ouest. Elle s'étend sur une superficie de 57735 hectares et est composée de 10 communes, avec une population estimée à 4395243 habitants (INS, 2014). Elle est en perpétuelle croissance et cette croissance est marquée par une forte urbanisation.

Sur le plan géologique, la lithologie de la ville est constituée de haut en bas de sables argileux, de sables moyens et de sables grossiers reposant sur un socle schisteux. Les formations schisteuses du socle sont constituées d'une part, par des roches granitiques (granite, granodiorite) et probablement des diorites quartziques (tonalites), et d'autre part, par des roches volcaniques, certainement calco-alcalines (Kouamé et al., 2006).

Les travaux de Kouakou et al. (2008) ont révélé que la ville d'Abidjan possède six importants sites de maraîchage (Figure 1). Notre étude a été menée sur la ferme expérimentale de l'Université Nangui Abrogoua (T) et les sites de maraîchage de Marcory (M) et de Cocody (C). 


\section{Stratégie d'échantillonnage des sols}

Les échantillonnages ont porté sur des parcelles ayant une superficie de $60 \mathrm{~m}$ x $45 \mathrm{~m}$. Les sols ont été prélevés sur des planches de semis (Figure 2), à l'aide d'une tarière, à une profondeur de $20 \mathrm{~cm}$ (horizon exploré par les racines des plantes maraîchères). Pour les analyses, ce sont 27 échantillons de sol qui ont été retenus pour chacun des sites de maraîchage (Marcory et Cocody) et 9 échantillons pour la parcelle expérimentale de l'université. Le nombre d'échantillons de sols analysés, plus élevé pour les sites maraîchers de Marcory et Cocody que celui de la ferme expérimentale de l'université, se justifie par l'hypothèse selon laquelle les sols de Marcory et Cocody seraient hétérogènes, compte tenu des pratiques agricoles alors que le sol témoin, qui n'a pas été l'objet d'un épandage de déchets, serait moins hétérogène.

\section{Analyse des sols}

Sur des échantillons de sols séchés et tamisés à $2 \mathrm{~mm}$, ont été effectués:

- des analyses classiques, à savoir, la granulométrie selon la méthode de la pipette de Robinson Kohl, le $\mathrm{pH}_{\text {eau }}$ en mettant en contact $20 \mathrm{~g}$ de sol et $50 \mathrm{~mL}$ d'eau distillée, avec agitation pendant $2 \mathrm{~h}$, la capacité d'échange cationique (CEC), avec le
$\mathrm{CH}_{3} \mathrm{COONH}_{4}$ tamponné à $\mathrm{pH} 7$, le carbone organique, selon la méthode de Walkley et Black (Mathieu et Piétain, 2003) basée sur l'oxydation de celui-ci par le $\mathrm{K}_{2} \mathrm{Cr}_{2} \mathrm{O}_{7}$ en présence de $\mathrm{H}_{2} \mathrm{SO}_{4}$;

- les dosages en éléments minéraux majeurs, comme le fer $(\mathrm{Fe})$ et le manganèse $(\mathrm{Mn})$, et en éléments traces métalliques, tels que le cadmium $(\mathrm{Cd})$, le cuivre $(\mathrm{Cu})$, le plomb $(\mathrm{Pb})$, le zinc $(\mathrm{Zn})$ et le nickel $(\mathrm{Ni})$. Pour ce faire, les sols ont été broyés et mis en solution par l'eau régale (mélange d'acide nitrique et d'acide chlorhydrique) selon la norme NF X31- 415 (AFNOR NF X31-415, 1995). Le dosage des métaux a été effectué au spectromètre d'absorption atomique.

\section{Traitement des données}

Les données recueillies ont été traitées au moyen du logiciel STATISTICA 6.0. L'analyse en composantes principales (ACP), qui est une méthode essentiellement descriptive, a été réalisée pour identifier les variables qui caractérisent chaque échantillon. Elle a porté sur l'ensemble des 63 échantillons (individus) provenant des horizons de surface des trois sites. Une variable contribue plus à la formation d'un axe lorsque la valeur absolue du coefficient de corrélation entre la variable et l'axe est supérieure ou égale à 0,70 .

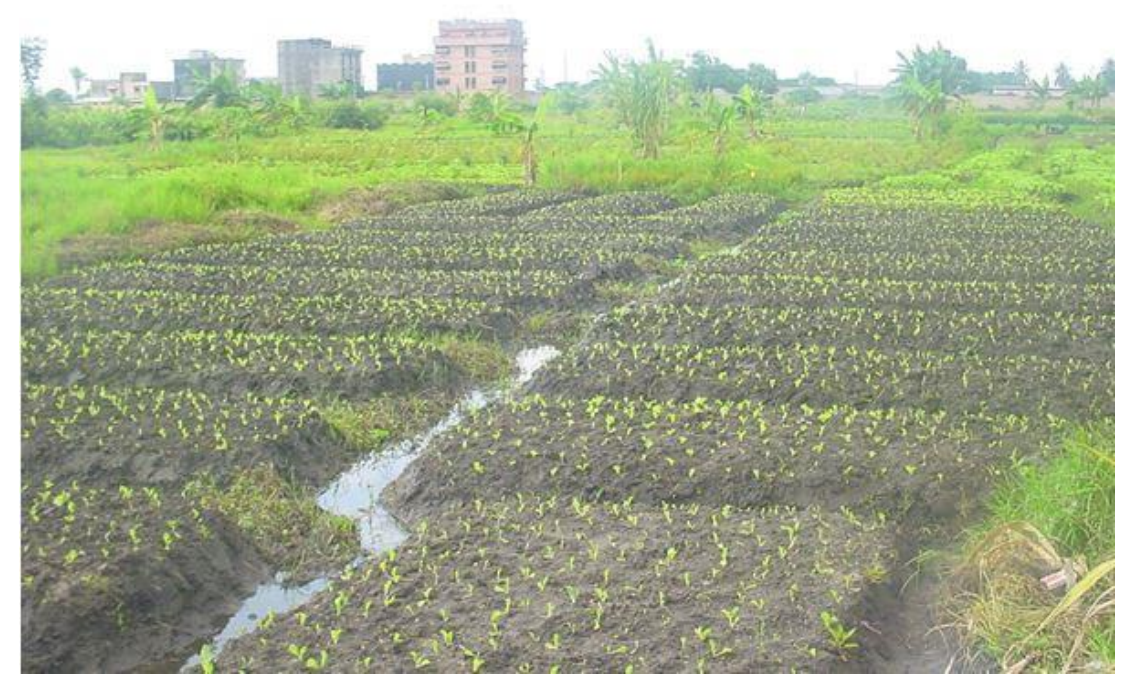

Figure 2 : Planches à laitues à Marcory. 

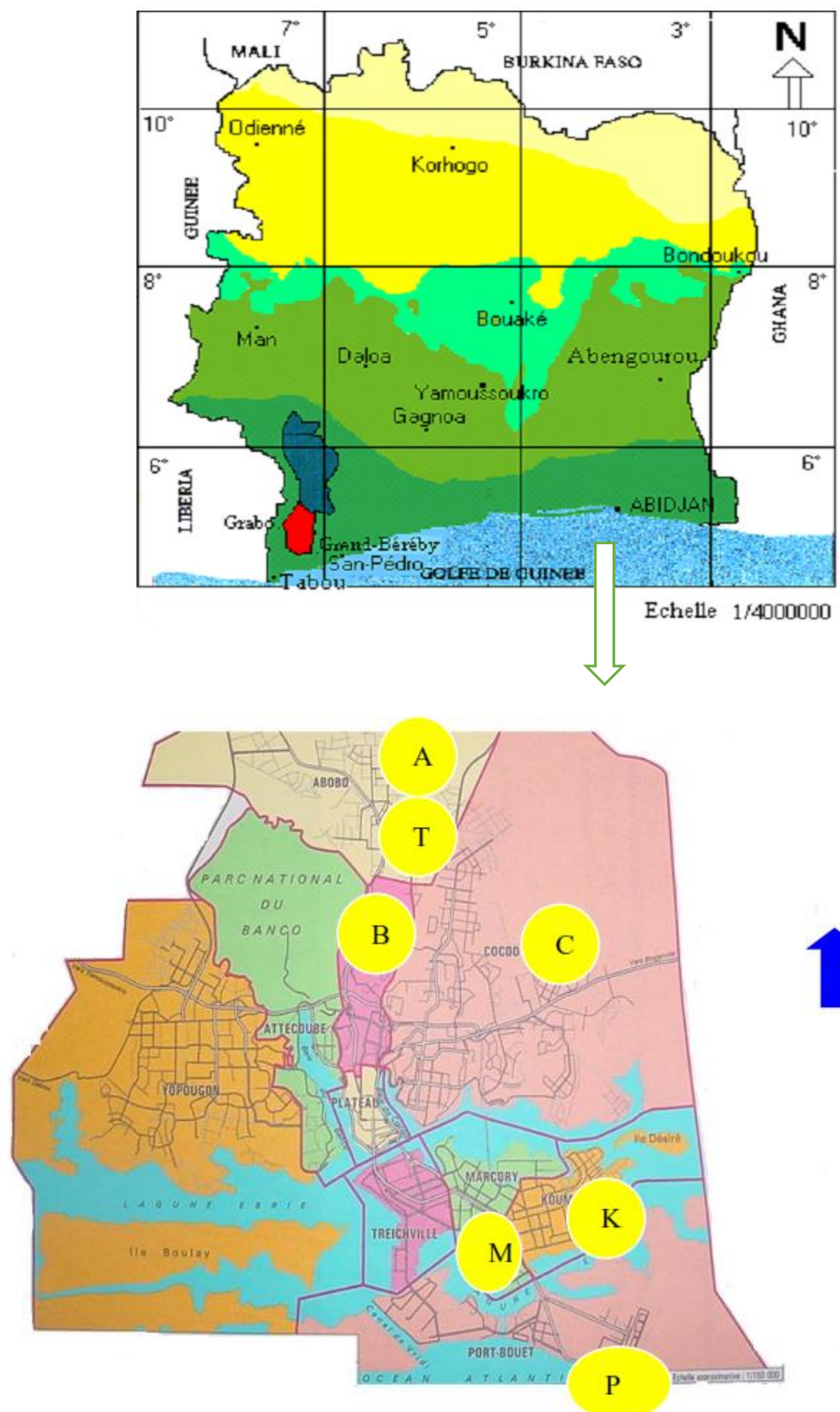

$\mathrm{A}=$ Abobo, $\mathrm{B}=$ Banco, $\mathrm{C}=$ Cocody, $\mathrm{K}=$ Koumassi, $\mathrm{M}=$ Marcory, $\mathrm{P}=$ Port-Bouët

Figure 1: Répartition des six importantes zones maraîchères au sein de l'agglomération abidjanaise (Kouakou et al., 2008). 


\section{RESULTATS}

L'interprétation des résultats a porté essentiellement sur les deux premières composantes principales (axes). Les valeurs propres de l'ACP indiquent que la première composante principale (axe 1) et la seconde (axe 2) expriment, respectivement, 32,56\% et $18,85 \%$ de l'inertie totale, soit un total de $51,41 \%$ (Tableau 1 ).

Le Tableau 1 et le graphique de la distribution des variables (Figure 3) indiquent que $\mathrm{Cu}, \mathrm{Zn}, \mathrm{Mn}$ et $\mathrm{Fe}$ sont fortement corrélés à l'axe 1 . Cu et $\mathrm{Zn}$ sont corrélés positivement à cet axe, tandis que $\mathrm{Mn}$ et Fe y sont corrélés négativement. Le
$\mathrm{pH}$ et le sable sont négativement et fortement corrélés à l'axe 2 .

A partir de la distribution des individus dans le plan 1-2 de l'ACP (Figure 4), l'on constate que les échantillons de sols sont différents selon leur origine. Ainsi, les horizons de surface des sols à Marcory sont caractérisés par $\mathrm{Zn}, \mathrm{Cu}$ et la matière organique. Les sols de Cocody sont caractérisés par $\mathrm{Ni}, \mathrm{Cd}$ et $\mathrm{Pb}$. A Cocody, trois échantillons tendent à se distinguer du reste des individus par leurs teneurs élevées en $\mathrm{Cd}$ et en $\mathrm{Pb}$. $\mathrm{Mn}, \mathrm{Fe}$, Ni et l'argile caractérisent les horizons de surface des sols du site témoin.

Tableau 1 : Matrice des valeurs propres, corrélations entre les variables et les deux axes révélés par l'ACP.

\begin{tabular}{lcc}
\hline \multirow{2}{*}{ Valeur propre } & Axe 1 & Axe 2 \\
\cline { 2 - 3 } & 4,23 & 2,45 \\
\hline Pourcentage de la variance totale & 32,56 & 18,85 \\
Pourcentage de la variance totale & 32,56 & 51,41 \\
cumulée & & \\
$\mathrm{Cd}$ & $-0,68$ & $-0,53$ \\
$\mathrm{Cu}$ & $\mathbf{0 , 7 8}$ & 0,12 \\
$\mathrm{~Pb}$ & $-0,28$ & $-0,58$ \\
$\mathrm{Zn}$ & $\mathbf{0 , 8 4}$ & 0,03 \\
$\mathrm{Ni}$ & $-0,63$ & $-0,19$ \\
$\mathrm{Carbone}$ organique (Corg) & 0,44 & $-0,09$ \\
$\mathrm{pH}$ & 0,25 & $\mathbf{- 0 , 7 0}$ \\
Capacité d'échange cationique & 0,38 & $-0,29$ \\
(CEC) & 0,13 & $\mathbf{- 0 , 7 6}$ \\
Sable & 0,21 & 0,66 \\
Limon & $-0,48$ & 0,16 \\
Argile & $\mathbf{- 0 , 8 3}$ & 0,14 \\
Mn & $\mathbf{- 0 , 7 7}$ & 0,37 \\
Fe & & \\
\hline
\end{tabular}

En gras : coefficients de corrélation significatifs 


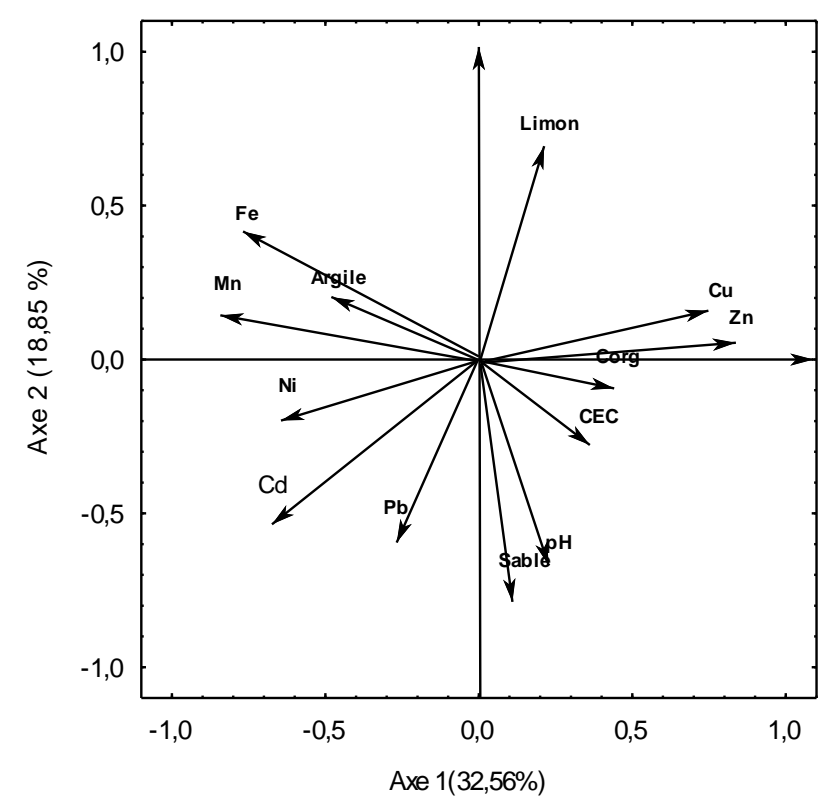

Figure 3: Distribution des variables dans le plan 1-2 de l'ACP portant sur les horizons de surface des sols.

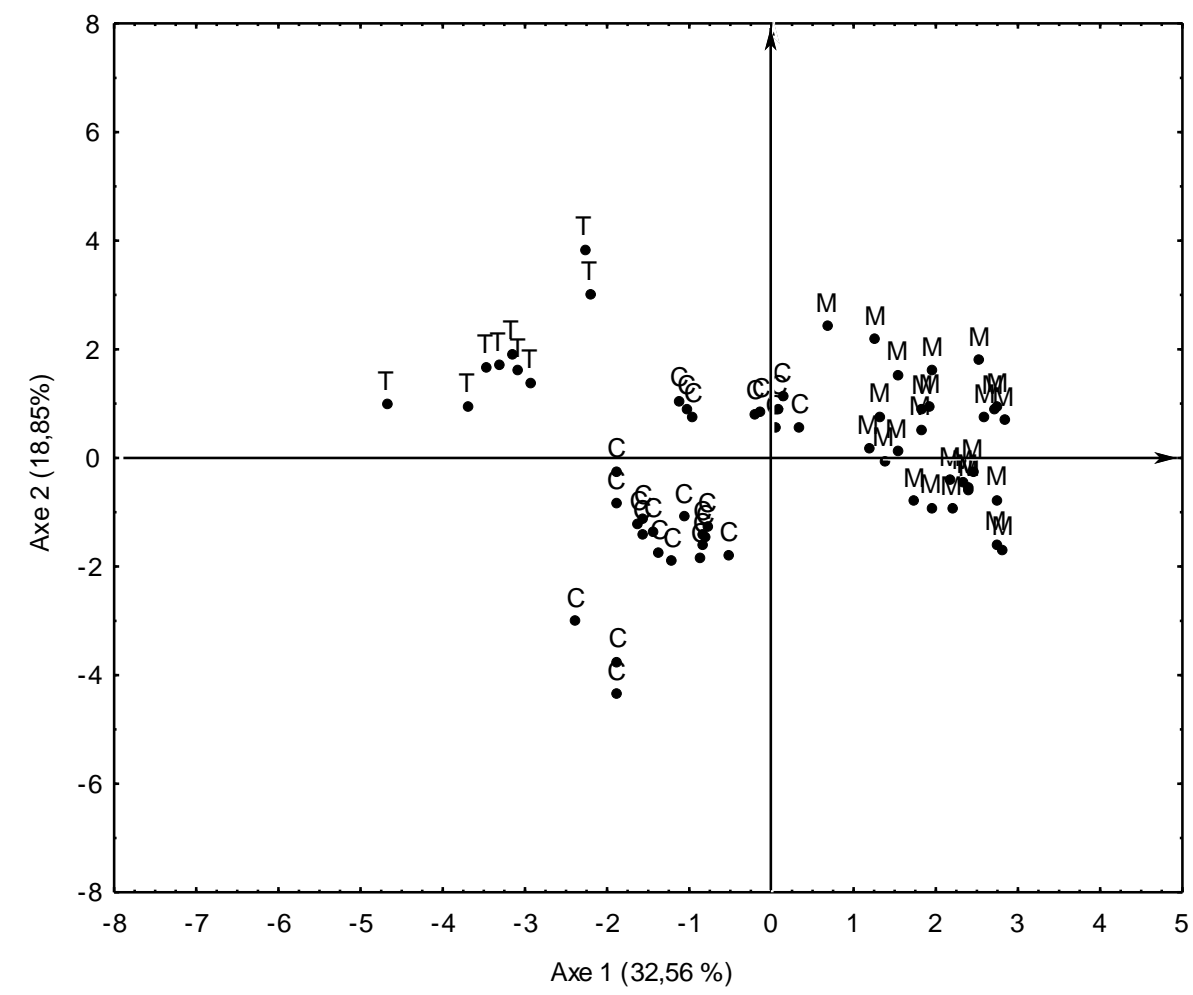

$\mathrm{M}=$ Marcory; $\mathrm{C}=$ Cocody; $\mathrm{T}$ = Parcelle expérimentale de l'Université Nangui Abrogoua

Figure 4: Distribution des échantillons de sol dans le plan 1-2 de l'ACP. 


\section{DISCUSSION}

L'ACP a permis de séparer les sites qui se distinguent les uns des autres. La différence entre les sols des trois sites serait imputable aux pratiques agricoles, telles que l'épandage de fiente de volaille (Kouakou et al., 2008) et les traitements phytosanitaires (Ehoussou, 2004), aux émissions atmosphériques et au matériau parental sur lequel chacun des sols s'est formé. En effet, la quantité de fiente de volaille épandue par les maraîchers varie selon les sites, et pour un même site, selon les planches de semis. Cette fumure non contrôlée, couplée aux divers traitements phytosanitaires, pourrait modifier inévitablement les propriétés des sols (teneurs en matière organique, teneurs en métaux traces, capacité d'immobilisation des métaux). A l'exception des métaux traces apportés par les pratiques agricoles, les sols urbains cultivés sont soumis à des pollutions abondantes en éléments traces dues aux retombées atmosphériques liées aux activités anthropiques (Laperche et Mosmann, 2004). Dans la ville d'Abidjan, les émissions atmosphériques concernent l'incinération des ordures, les activités artisanales du travail des métaux, le trafic routier et les activités industrielles. En temps de pluie, les sites de Marcory et de Cocody sont des réceptacles privilégiés des eaux de ruissellement qui constituent, aussi, une autre source d'éléments traces. L'héritage géopédologique, dont les données sont absentes dans la littérature, est un paramètre qui pourrait influer sur les teneurs des sols en éléments traces (SchmittSirguey, 2004). Nos résultats sont similaires à ceux des travaux de Adjia et al. (2008). En effet, ces auteurs ont montré que les sols de trois sites maraîchers et un site témoin à Ngaoundéré, au Cameroun, étaient dissemblables pour les concentrations en $\mathrm{Cd}$, $\mathrm{Cu}, \mathrm{Ni}, \mathrm{Pb}$ et $\mathrm{Zn}$. Ils ont attribué cette variation des teneurs en métaux traces entre les sites à l'importance des activités anthropiques autour de ces sites, susceptibles de générer les déchets urbains. Ces déchets urbains apportent surtout $\mathrm{Cu}, \mathrm{Zn}$ et $\mathrm{Pb}$ aux sols agricoles (Feix et Wiart, 1998).

\section{Conclusion}

Il ressort de notre étude que les sols à maraîchers de Marcory et Cocody et le sol de la parcelle expérimentale de l'Université Nangui Abrogoua sont caractérisés par des paramètres agro-pédologiques (texture, $\mathrm{pH}$, capacité d'échange cationique, teneur en matière organique, teneurs en $\mathrm{Fe}$ et $\mathrm{Mn}$ ) et d'éléments traces métalliques $(\mathrm{Cd}, \mathrm{Cu}, \mathrm{Pb}, \mathrm{Zn}$, $\mathrm{Ni})$ différents. Ces différences ont été attribuées aux pratiques agricoles non contrôlées, aux activités urbaines, sources d'éléments traces, et au matériau parental sur lequel chacun des sols s'est formé. Pour compléter cette étude, il est prévu des tests de biodisponibilité des métaux traces pour les plantes cultivées sur ces sites.

\section{REMERCIEMENTS}

Les auteurs remercient l'UNESCO, le Gouvernement japonais, la Fondation Internationale pour la Science (FIS) et l'Organisation d'Interdiction des Armes Chimiques (OIAC) qui ont financé cette étude.

\section{RÉFÉRENCES}

Adjia R, Fezeu WML, Tchatchueng JB, Sorho S, Echevarria G, Ngassoum MB. 2008. Long term effect of municipal solid waste amendment on soil heavy metal content of sites used for periurban agriculture in Ngaoundere, Cameroon. African Journal of Environmental Science and Technology, 2(12): 412-421.

AFNOR NF X31-415. 1995. Qualité des sols, extraction des éléments en traces solubles dans l'eau régale, Paris, 455463.

Baize D. 2000. Teneurs totales en "métaux lourds" dans les sols français, résultats généraux du programme ASPITET. Courrier de l'Environnement de l'INRA, 39 : $39-54$.

CLUB CRIN. 1999. Spéciation des métaux dans le sol, Association ECRIN, 296 p.

Ehoussou KM. 2004. Contribution à l'étude de la contamination des produits maraîchers par les résidus de pesticides: cas de la ville d'Abidjan. Diplôme 
d'Etudes Approfondies, Université d'Abobo-Adjamé, Côte d'Ivoire, $54 \mathrm{p}$.

Feix I, Wiart J. 1998. Connaissance et Maîtrise des Aspects Sanitaires de l'Epandage des Boues d'Epuration des Collectivités Locales. Ed. ADEME: Angers, France ; 74p.

INS (Institut National de la Statistique). 2014. Recensement Général de la Population et de l'Habitat. Rapport d'exécution et présentation des principaux résultats. 49 p.

Kouakou KJ, Bekro YA, Sika AE, Baize D, Dogbo DO, Bounakhla M, Zahary F, Macaigne P. 2008. Diagnostic d'une contamination par les éléments traces métalliques de l'épinard (Spinacia oleracea) cultivé sur des sols maraîchers de la ville d'Abidjan (Côte d'Ivoire) amendés avec de la fiente de volaille. European Journal of Sciences Research, 21 (3) : 471-487.
Kouamé KI, Goné DL, Savané I , Kouassi EA, Koffi K, Goula BT et Diallo M. 2006. Mobilité relative des métaux lourds issus de la décharge d'Akouédo et risque de contamination de la nappe du Continental Terminal (Abidjan-Côte d'Ivoire). Afrique Sciences, 2 (1) : 39-56.

Laperche V, Mosmann JR. 2004. Protocoles d'échantillonnage des sols pollués par du plomb.RP-52928-FR, 26 p.

Mathieu C, Piétain F. 2003. Analyse Chimique des Sols : Méthodes Choisies. Édition Tec. et Doc. ; 387 p.

Schmitt-Sirguey C. 2004. Dynamique du Cd disponible du sol sous l'influence de l'hyperaccumulateur Thlaspi caerulenscens, Thèse de Doctorat de l'Institut National Polytechnique de Lorraine, $157 \mathrm{p}$.

Tremel-Schaub A, Feix I. 2005. Contamination des Sols : Transferts des Sols vers les Plantes. EDP Sciences, ADEME ; 416 p. 